\title{
Penicillin G-Induced Chlamydial Stress Response in a Porcine Strain of Chlamydia pecorum
}

\author{
Cory Ann Leonard, ${ }^{1}$ Frederic Dewez, ${ }^{2}$ and Nicole Borel ${ }^{1}$ \\ ${ }^{1}$ Department of Pathobiology, Institute of Veterinary Pathology, University of Zurich, Winterthurerstrasse 268, \\ 8057 Zurich, Switzerland \\ ${ }^{2}$ University of Lille-Sciences and Technologies, Cite Scientifique, Villeneuve d'Ascq Cedex, 59655 Lille, France
}

Correspondence should be addressed to Nicole Borel; n.borel@access.uzh.ch

Received 2 December 2015; Accepted 26 January 2016

Academic Editor: Todd R. Callaway

Copyright (c) 2016 Cory Ann Leonard et al. This is an open access article distributed under the Creative Commons Attribution License, which permits unrestricted use, distribution, and reproduction in any medium, provided the original work is properly cited.

Chlamydia pecorum causes asymptomatic infection and pathology in ruminants, pigs, and koalas. We characterized the antichlamydial effect of the beta lactam penicillin G on Chlamydia pecorum strain 1710S (porcine abortion isolate). Penicillinexposed and mock-exposed infected host cells showed equivalent inclusions numbers. Penicillin-exposed inclusions contained aberrant bacterial forms and exhibited reduced infectivity, while mock-exposed inclusions contained normal bacterial forms and exhibited robust infectivity. Infectious bacteria production increased upon discontinuation of penicillin exposure, compared to continued exposure. Chlamydia-induced cell death occurred in mock-exposed controls; cell survival was improved in penicillinexposed infected groups. Similar results were obtained both in the presence and in the absence of the eukaryotic protein translation inhibitor cycloheximide and at different times of initiation of penicillin exposure. These data demonstrate that penicillin $\mathrm{G}$ induces the chlamydial stress response (persistence) and is not bactericidal, for this chlamydial species/strain in vitro, regardless of host cell de novo protein synthesis.

\section{Introduction}

The family Chlamydiaceae comprises Gram negative, obligate intracellular bacterial pathogens of humans and wild and domestic animals. In humans, C. trachomatis causes trachoma and blindness as well as genital tract infection and infertility, while C. pneumoniae seropositivity is highly prevalent and infection can be associated with respiratory disease and atherosclerosis. C. psittaci and C. abortus are important pathogens of birds and farm animals and are capable of zoonotic transmission to humans. C. pecorum is generally accepted as an important pathogen of cattle, sheep, and especially koalas [1]. Symptoms associated with C. pecorum infection include polyarthritis, pneumonia, encephalomyelitis, and abortion in livestock and conjunctivitis, urinary/reproductive disease, and infertility in koalas [1]. In pigs, C. pecorum has been associated with pneumonia, enteritis, polyarthritis, pericarditis/pleuritis, and urogenital infections, as well as asymptomatic infections [2].
The C. pecorum strain $1710 \mathrm{~S}$ was originally isolated in Austria from a swine abortion, in association with a large outbreak of chlamydiosis of varying clinical presentation $[3,4]$. C. pecorum $1710 \mathrm{~S}$ experimentally infected gnotobiotic piglets demonstrated mild to moderate diarrhea and a transient stop of weight gain; these symptoms were associated with mild to moderate villus atrophy and mixed inflammatory cell infiltrates in the small intestinal mucosa [5]. C. pecorum $1710 \mathrm{~S}$ was also implicated, by sequence homology of cloned Chlamydia omp1 genus-specific PCR products, in 8 of 9 Chlamydia-positive aborted fetuses of Swiss swine $[6,7]$, emphasizing the pathogenic potential of this isolate. More recently, 51 female calves in the US, evaluated from birth to 6 months, all became C. pecorum-positive by $23 \mathrm{~S}$ rRNA PCR and ompA genotyping of conjunctival and vaginal swabs; $C$. pecorum 1710S was 1 of the 3 C. pecorum strains identified by PCR product sequencing [8]. Interestingly, in this study, C. pecorum infections were associated with failure to gain weight and increased conjunctival redness but not intestinal 
symptoms or other diseases [8]. Taken together, these data indicate that $C$. pecorum $1710 \mathrm{~S}$ has pathogenic potential and/or can lead to decrease of animal weight gain in both swine and cattle.

In livestock, infections are routinely treated with antibiotics, especially tetracyclines [9]. However, the use of tetracyclines has been shown to be both ineffective in eradicating chlamydial infections in swine [10] and associated with the presence of tetracycline-resistant C. suis [11]. Beta lactam antibiotics, such as penicillin and amoxicillin, are widely used for various infections, and their persistence in animal tissue is a particular concern in meat and dairy animals, largely due to possible dangerous effects in penicillin-allergic individuals $[12,13]$. In swine specifically, penicillin $G$ has been detected in various tissues/organs, especially the kidney, for up to 39 days after injection and is expected to persist in detectable amounts for up to 47 days $[14,15]$. C. pecorum infections in pigs are frequently asymptomatic and/or are not diagnosed because they are considered unimportant pathogens in swine [2]. At the same time, the use of both veterinary-approved and off-label antibiotics is widespread in pig production. Therefore, it is likely that pigs asymptomatically infected with C. pecorum are treated with antibiotics administered for other purposes.

In the more well-studied Chlamydiae, such as C. trachomatis, C. muridarum, C. pneumoniae and C. psittaci, beta lactam antibiotics, such as penicillin and ampicillin, have historically and consistently been demonstrated to be ineffective against eradicating chlamydial infection, both in vitro and in animal models [16-22]. Instead of killing the Chlamydiae, beta lactams induce persistence or the chlamydial stress response, defined as a viable but noninfectious divergence from the normal chlamydial biphasic developmental cycle [23]. Persistent/stressed Chlamydiae are less susceptible to killing by antibiotics such as azithromycin [21, 24], undetectable by culture, and capable of resuming infectious bacteria production upon removal of the stressor/antibiotic [25].

Information about the efficacy of antibiotics against $C$. pecorum specifically, however, is very limited. A single in vitro study of 3 calf and lamb $C$. pecorum isolates indicated that macrolides, tetracyclines, and quinolones, but not the beta lactam ampicillin, prevented inclusion formation; however, $\mathrm{AB}$ induction, infectious bacterial production and potential recovery upon removal of ampicillin were not evaluated [26]. More recently, 10 koala $C$. pecorum isolates were evaluated for susceptibility to enrofloxacin, chloramphenicol, and florfenicol, but beta lactam antibiotics were not considered [27]. Thus, the ability of beta lactam antibiotics to reversibly abrogate C. pecorum infectivity, by definition inducing chlamydial stress/persistence, has not been demonstrated. The aim of our study is characterization of the in vitro antichlamydial effect of the beta lactam penicillin $G$ on the $C$. pecorum porcine abortion strain $1710 \mathrm{~S}$, a potentially significant pathogen of swine and other economically important animals.

Our data indicate that the effect of penicillin $G$ on $C$. pecorum $1710 \mathrm{~S}$ is consistent with the definition of chlamydial stress/persistence. Specifically, persistence/chlamydial stress is characterized by reversible reduced or abolished infectious bacteria production and aberrant, enlarged bacterial forms called aberrant bodies (AB) detectable by immunofluorescence and transmission electron microscopy [25, 28-30]. We further determine that, under the experimental conditions used herein, penicillin $G$ induces chlamydial stress in $C$. pecorum 1710S regardless of inclusion/exclusion of cycloheximide (which limits host de novo protein synthesis), though recovery is more robust in the presence of cycloheximide. Finally, initiation of penicillin exposure at 0 or 14 hours postinfection (hpi) yields equivalent reductions of infectious EB production and subsequent recovery of infectious EB production.

\section{Materials and Methods}

2.1. Host Cells. HeLa cells (human cervical adenocarcinoma epithelial cells, CCL-2, American Type Culture Collection, Manassas, VA, USA) were cultured for cell propagation and maintenance at $37^{\circ} \mathrm{C}$ and $5 \% \quad \mathrm{CO}_{2}$ in growth medium. Growth medium was comprised of Minimal Essential Medium (MEM) with Earle's salts, $25 \mathrm{mM}$ HEPES, without L-Glutamine (GIBCO, Invitrogen, Carlsbad, CA, USA) supplemented with $10 \%$ fetal calf serum (FCS, BioConcept, Allschwil, Switzerland), $4 \mathrm{mM}$ GlutaMAX-I (200 mM, GIBCO), 1\% MEM Nonessential Amino Acids $(100 \mathrm{x}, \mathrm{GIBCO})$, and $0.2 \mathrm{mg} / \mathrm{mL}$ gentamycin $(50 \mathrm{mg} / \mathrm{mL}$, GIBCO). For experiments, cells were seeded in 24-well plates (Techno Plastic Products AG (TPP), Trasadingen, Switzerland) at $3 \times 10^{5}$ cells per well in $1 \mathrm{~mL}$ growth medium without gentamycin. Cells were seeded on $13 \mathrm{~mm}$ diameter glass coverslips (Sterilin Limited, Thermo Fisher Scientific, Cambridge, UK) for immunofluorescence (IF) microscopy or transmission electron microscopy (TEM) or directly in the 24-well plates, without coverslips, for titration by subpassage. Infection medium, used for inoculating cells with Chlamydia, consisted of all growth medium components except FCS and gentamycin. Incubation medium, used to replace infection medium after inoculation with Chlamydia, consisted of all growth medium components except gentamycin and was further supplemented with $1 \mu \mathrm{g} / \mathrm{mL}$ cycloheximide (SigmaAldrich, St. Louis, MO, USA) immediately before use. In some experiments, growth medium without cycloheximide supplementation was used for incubation.

2.2. Chlamydial Strain. C. pecorum 1710 S (isolate from a swine abortion) was kindly provided by Professor Storz, Baton Rouge, LA, USA [3] and propagated in HeLa cells. Crude C. pecorum stock was generated by mechanical disruption (scraping) of infected HeLa cells into infection medium, sonication (Branson Sonifier 250; Branson Ultrasonics, Danbury, CT, USA) on ice, centrifugation of infectious particles from the medium at $10,000 \mathrm{~g}$ at $4^{\circ} \mathrm{C}$ for 45 minutes, and suspension in SPG medium. Stock was stored at $-80^{\circ} \mathrm{C}$ and frozen stock aliquots were thawed immediately before infections were carried out. SPG medium consisted of $218 \mathrm{mM}$ sucrose (Sigma-Aldrich), $3.76 \mathrm{mM} \mathrm{KH}_{2} \mathrm{PO}_{4}$ (SigmaAldrich), $7.1 \mathrm{mM} \mathrm{K} \mathrm{HPO}_{4}$ (Merck Eurolab AG, Dietlikon, Switzerland), and $5 \mathrm{mM}$ GlutaMAX-100 (GIBCO). 


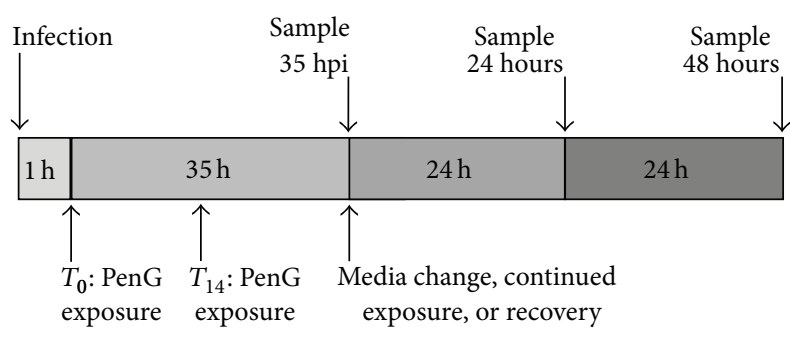

FIgURE 1: Study Design. The diagram illustrates infection and exposure beginning at $T_{0}$, immediately postinfection, or at $T_{14}, 14$ hours postinfection (hpi). HeLa cells were infected with Chlamydia pecorum 1710 S, exposed to penicillin $\mathrm{G}$ (PenG) in incubation medium, and incubated until $35 \mathrm{hpi}$, at which time samples were collected for analysis. For recovery experiments, incubation medium was changed for continued PenG exposure or discontinued exposure (recovery), and samples were subjected to 24 or 48 hours (h) of additional incubation before sample collection.

2.3. Penicillin Reagent. Penicillin G sodium salt (SigmaAldrich) was dissolved in sterile deionized water to a stock concentration of 20,000 units (U)/mL, filter-sterilized, and stored at $-20^{\circ} \mathrm{C}$. Aliquots of this penicillin G stock were thawed and further diluted in sterile water to a working concentration of $100 \mathrm{U} / \mathrm{mL}$ immediately before use. Working solutions were stored for less than one week at $4^{\circ} \mathrm{C}$, as per manufacturer's recommendation. The final penicillin $\mathrm{G}$ concentration used in experiments, $1 \mathrm{U} / \mathrm{mL}$, was achieved by $10 \mu \mathrm{L} / \mathrm{mL}$ dilution of working solution in the incubation medium of penicillin-exposed samples. Sterile water (diluent) of an equivalent volume was similarly used to generate control (mock-exposed) samples.

2.4. Study Design, Infection, and Exposure of Infected Host Cells to Penicillin. HeLa cells were cultivated overnight in 24well plates and were subsequently infected with C. pecorum $1710 \mathrm{~S}$ at 1 multiplicity of infection (MOI) in $1 \mathrm{~mL}$ infection medium per well and centrifuged for 1 hour (h) at $1000 \mathrm{~g}$ and $25^{\circ} \mathrm{C}$. After centrifugation, infection medium was immediately replaced with incubation medium and cultures were incubated at $37^{\circ} \mathrm{C}$ and $5 \% \mathrm{CO}_{2}$ as previously described [31]. The crude stocks used resulted in approximate control infection rates of $50 \%$; this corresponded to approximately $10^{7}$ recoverable inclusion-forming units (IFU) per well for mockexposed infected cells after 35 hours of incubation. The effect of penicillin $\mathrm{G}$ on $C$. pecorum inclusions was determined by adding penicillin $G$ to a final concentration of $1 \mathrm{U} / \mathrm{mL}$, to the incubation medium of infected cells at $T_{0}$, immediately after infection, or at $T_{14}, 14$ hours after infection (Figure 1). In experiments evaluating recovery from penicillin $\mathrm{G}$, incubation medium was changed at 35 hours postinfection (hpi) to continue exposure or discontinue exposure (recovery) to penicillin. This time, $35 \mathrm{hpi}$, corresponds to the presence of mature C. pecorum $1710 \mathrm{~S}$ inclusions in HeLa culture, but no significant host cell lysis, as previously reported [32].

At the indicated times, samples were collected and processed for further analysis as previously described [31]. For immunofluorescence (IF) microscopy, cells were fixed with absolute methanol $\left(-20^{\circ} \mathrm{C}\right)$ for 10 minutes. For transmission electron microscopy (TEM), cells were fixed with $2.5 \%$ glutaraldehyde (Electron Microscopy Sciences, Fort Washington, USA) for $1 \mathrm{~h}$ and embedded in epoxy resin (Fluka; SigmaAldrich) by routine methods. For titration by subpassage infected monolayers collected at $35 \mathrm{hpi}$ were scraped into $1 \mathrm{~mL}$ of fresh infection medium and stored at $-80^{\circ} \mathrm{C}$. Because C. pecorum 1710S-infected HeLa cells undergo significant lysis and EB release soon after $35 \mathrm{hpi}$, as previously reported [32], for continued exposure or recovery sample collection for titration by subpassage, infected monolayers were scraped into the existing incubation medium and stored at $-80^{\circ} \mathrm{C}$. In all experiments, biological duplicates were generated for each experimental condition.

2.5. IF Microscopy. C. pecorum inclusions were visualized using 1:200 diluted Chlamydiaceae family-specific mouse monoclonal antibody directed against the chlamydial lipopolysaccharide (LPS, Clone ACI-P; Progen, Heidelberg, Germany) and 1:500 diluted Alexa Fluor 488-conjugated secondary goat anti-mouse antibody (Molecular Probes, Eugene, OR, USA). Host and chlamydial DNA were labeled with $1 \mu \mathrm{g} / \mathrm{mL} 4^{\prime}, 6$-diamidino-2' -phenylindole dihydrochloride (DAPI, Molecular Probes). Coverslips were mounted with FluoreGuard mounting medium (Hard Set; ScyTek Laboratories Inc., Logan, UT, USA) on glass slides. Slides were evaluated using a Leica DMLB fluorescence microscope (Leica Microsystems, Wetzlar, Germany) under oil immersion at 1000x magnification with a 1006 objective (PL FLUOTAR 100x/1.30, OIL, $\infty / 0.17 / D$, Leica Microsystems) and a 106 ocular objective (Leica L-Plan 10x/25 M, Leica Microsystems). To determine percent of cells infected (inclusions per nucleus) and mean number of nuclei per field (to evaluate possible cell loss from the monolayer), HeLa nuclei and corresponding chlamydial inclusions in each of 10 randomly selected microscopic fields were counted per duplicate coverslip (at least $200 \mathrm{HeLa}$ nuclei per coverslip), and the mean of coverslips values was generated for each experimental condition. Representative microscopic images were captured using BonTec software (BonTec) and a UI2250SE-C-HQ camera (uEye, IDS Imaging Development Systems GmbH, Obersulm, Germany).

2.6. Chlamydial Titration by Subpassage. HeLa cells were grown on glass coverslips as described above. Immediately prior to use, the previously prepared and frozen samples were thawed and sonicated on ice for 5 minutes. Sonicated samples were serially diluted in infection medium on the prepared HeLa cells. Centrifugation, infection medium replacement with incubation medium (containing $1 \mu \mathrm{g} / \mathrm{mL}$ cycloheximide for all experiments), and incubation were carried out as described for infection of host cells. Fixation and immunostaining were performed exactly as described for IF microscopy. The number of inclusions in 30 random microscopic fields per duplicate coverslip was counted using a Leica fluorescence microscope at 200x magnification with a 206 objective (PL FLUOTAR 20x/0.50 PH 2, $\infty / 0.17 / \mathrm{B}$ ) and a 106 ocular objective (Leica L-Plan 10x/25 M, Leica Microsystems), and the mean of coverslips values was 

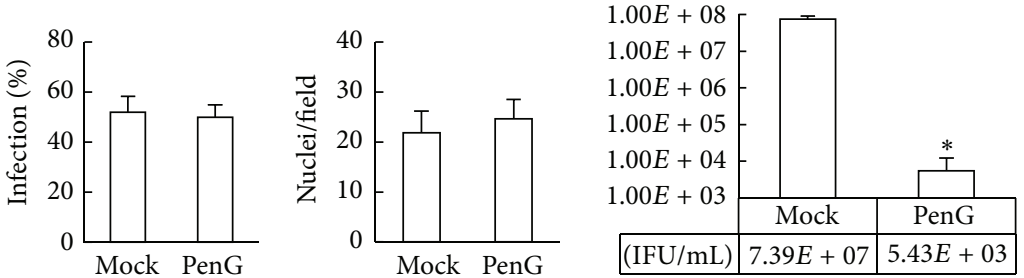

(a)
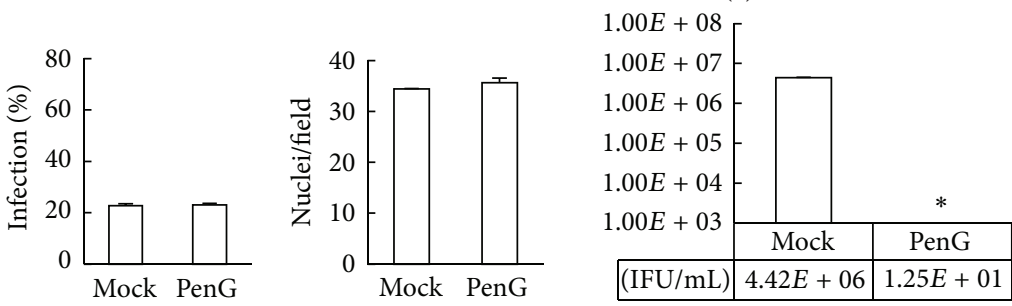
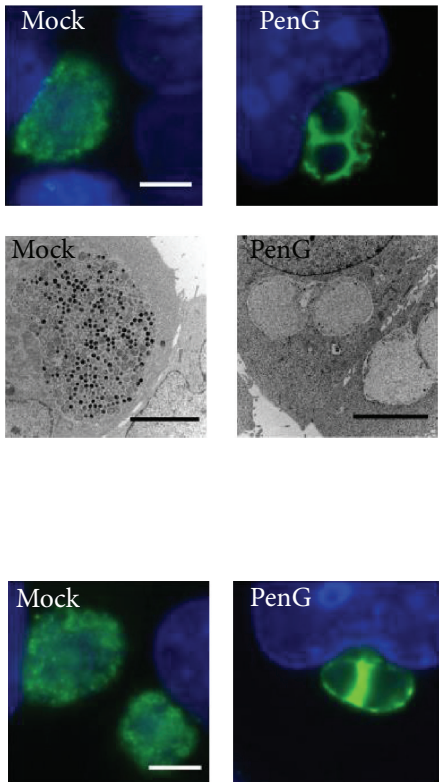

(b)

FIGURE 2: Penicillin G exposure induces aberrant body formation and reduces infectious elementary body production in Chlamydia pecorum. C. pecorum-infected HeLa cells were exposed to penicillin G (PenG), at 0 hours postinfection (hpi) until 35 hpi. Control infected cells were exposed to diluent only (mock). Cycloheximide was included (a) or not included (b) in the incubation medium. Inclusions were visualized using an antibody directed against chlamydial lipopolysaccharide (green), and DNA was labeled with $4^{\prime}, 6$-diamidino- $2^{\prime}$-phenylindole dihydrochloride (blue). Percent of host cells infected and mean nuclei per field was determined by evaluation of 10 fields ( $\geq 200$ cells), per coverslip, per sample. Representative immunofluorescence and transmission electron microscopic images illustrate normal inclusions in mock-exposed controls and inclusions containing aberrant bodies upon penicillin exposure. Infectious elementary body production was determined by titration by subpassage and expressed as inclusion-forming units (IFU)/mL. Results are means \pm standard deviation. The two-tailed $t$-test was used to compare means; $p \leq 0.05=$ significant $\left({ }^{*}\right) ; n=3$ (a) or $n=2$ (b). Scale bars $=5 \mu \mathrm{m}$.

generated for each experimental condition. IFU/mL of undiluted inoculum (representing IFU per total collected material from a single well of a 24 -well plate) was calculated according to previously published methods [33].

2.7. TEM. Ultrathin $(80 \mathrm{~nm})$ sections were mounted on gold grids (Merck) and contrasted with uranyl acetate dehydrate (Fluka; Sigma-Aldrich) and lead citrate (Merck). Sections were subsequently evaluated using a Philips CM10 electron microscope (Software release version 5.1; FEI Company, Hillsboro, OR, USA) and imaged using a Gatan Orius SC 1000 CCD Camera with software version Digital Micrograph 2.30 (Gatan Inc., Warrendale, PA, USA). All images were analyzed using Photoshop CS6 software (Adobe Systems Incorporated, San Jose, CA, USA). To determine the presence of $A B$, bacterial morphology analysis was carried out, as previously described [34]: EB (dark, 0.25-0.5 $\mu \mathrm{m}$ ), IB (intermediate bodies; dark center and pale periphery, equivalent in size to $\mathrm{EB}$ or $\mathrm{RB}$ ), RB (pale, $0.5-1 \mu \mathrm{m}$ ), and $\mathrm{AB}$ (pale, $\geq 2 \mu \mathrm{m}$ ).

2.8. Statistical Analysis. In all experiments, biological duplicates were averaged to generate results. Statistical analyses were performed using Microsoft Excel. Significance of the difference of means was determined by unpaired $t$-test and $p$ values of $<0.05$ were considered significant. $p$ values were confirmed using the GraphPad QuickCalcs Web site: http://www.graphpad.com/quickcalcs/ttestl/ (accessed June 2014). Unless stated otherwise, results are displayed as means \pm standard deviation, of the results from 2 or 3 independent experiments. In cases where a single confirmatory experiment is noted, results are displayed as means \pm standard deviation, of the two biological duplicates.

\section{Results and Discussion}

In this study we evaluated the effect of the beta lactam antibiotic penicillin $\mathrm{G}$ on a porcine abortion strain of Chlamydia pecorum (1710S). Based on previous reports that various chlamydial species enter chlamydial stress/persistence upon beta lactam exposure [16-22], we hypothesized that penicillin $\mathrm{G}$ would induce chlamydial stress in C. pecorum $1710 \mathrm{~S}$. Because inclusion/exclusion of the eukaryotic protein synthesis inhibitor cycloheximide has the potential to influence the effect of beta lactams on Chlamydiae [35], we evaluated the effect of penicillin G on C. pecorum in the presence of cycloheximide and performed a single confirmatory experiment in the absence of cycloheximide.

HeLa cells were infected with $C$. pecorum, exposed to penicillin $\mathrm{G}$ or sterile water (mock-exposed control) in the cycloheximide-containing or cycloheximide-free incubation medium at 0 hours postinfection (hpi), and incubated until 35 hpi (Figure 1). For cycloheximide-exposed HeLa/C. pecorum (Figure $2(\mathrm{a})$ ), infection rates of $50-52 \%$ of diluent- or 
TABLE 1: Chlamydia pecorum recovers infectious elementary body production (inclusion-forming units/mL) upon discontinuation of penicillin G exposure.

\begin{tabular}{|c|c|c|c|c|c|}
\hline Hours & Experimental group & Experiment 1 & $p$ & Experiment 2 & $p$ \\
\hline \multirow{5}{*}{24} & Mock & $1.21 \times 10^{8} \pm 2.20 \times 10^{6}$ & & $5.11 \times 10^{7} \pm 1.21 \times 10^{6}$ & \\
\hline & PenG 0 hpi & $3.25 \times 10^{1} \pm 1.77 \times 10^{1}$ & $0.0003^{\#}$ & $1.00 \times 10^{1} \pm 7.07 \times 10^{0}$ & $0.0002^{\#}$ \\
\hline & PenG 0 hpi recovery & $1.77 \times 10^{5} \pm 1.00 \times 10^{4}$ & $0.0016^{\# \#}$ & $6.36 \times 10^{3} \pm 2.75 \times 10^{3}$ & $0.0825^{\# \#}$ \\
\hline & PenG 14 hpi & $1.75 \times 10^{1} \pm 3.54 \times 10^{0}$ & $0.0003^{\#}$ & $3.00 \times 10^{1} \pm 1.41 \times 10^{1}$ & $0.0002^{\#}$ \\
\hline & PenG 14 hpi recovery & $1.63 \times 10^{5} \pm 1.40 \times 10^{4}$ & $0.0037^{\# \#}$ & $1.52 \times 10^{4} \pm 2.13 \times 10^{4}$ & $0.4196^{\# \#}$ \\
\hline \multirow{5}{*}{48} & Mock & $4.89 \times 10^{7} \pm 2.73 \times 10^{7}$ & & $2.73 \times 10^{7} \pm 9.06 \times 10^{5}$ & \\
\hline & PenG 0 hpi & $5.50 \times 10^{1} \pm 2.83 \times 10^{1}$ & $0.0026^{\#}$ & $3.00 \times 10^{1} \pm 7.07 \times 10^{0}$ & $0.0019^{\#}$ \\
\hline & PenG 0 hpi recovery & $1.66 \times 10^{6} \pm 1.18 \times 10^{5}$ & $0.1345^{\# \#}$ & $1.02 \times 10^{7} \pm 5.62 \times 10^{5}$ & $0.0015^{\# \#}$ \\
\hline & PenG 14 hpi & $1.28 \times 10^{2} \pm 3.54 \times 10^{0}$ & $0.1314^{\#}$ & $6.75 \times 10^{1} \pm 3.54 \times 10^{0}$ & $0.0249^{\#}$ \\
\hline & PenG 14 hpi recovery & $9.69 \times 10^{5} \pm 6.02 \times 10^{3}$ & $<0.0001^{\# \#}$ & $6.11 \times 10^{6} \pm 4.73 \times 10^{6}$ & $0.2091^{\# \#}$ \\
\hline
\end{tabular}

C. pecorum 1710S-infected HeLa cells were exposed to diluent only (mock) or to penicillin G at 0 hours postinfection (hpi) or $14 \mathrm{hpi}$. At $35 \mathrm{hpi}$, medium was changed and exposure was continued or discontinued (recovery) for 24 or 48 hours. Inclusion counts generated from biological duplicates were used to calculate mean inclusion-forming units/mL. Results from 2 independent experiments evaluating $24 \mathrm{~h}$ of recovery or $48 \mathrm{~h}$ of recovery are shown. $p$ values for groups compared to corresponding mock (\#) or corresponding continued exposure groups (\#\#) are shown; $p \leq 0.05=$ significant.

0 hpi penicillin-exposed cells were observed. Mean nuclei per field values were 22 and 25 for diluent- and 0 hpi penicillinexposed infected cells, respectively. When cycloheximide was omitted (Figure 2(b)), an infection rate of $23 \%$ was observed for mock- and 0 hpi penicillin-exposed cells. In the absence of cycloheximide, mean nuclei per field values were 35 for mock-exposed cells and 36 for 0 hpi penicillinexposed cells. Thus, penicillin G had no effect on host cell infection rate or host cell loss from the monolayer at $35 \mathrm{hpi}$, regardless of time of initiation of penicillin exposure, whether or not cycloheximide was included. However, as expected, the omission of cycloheximide allowed host cell division to continue and resulted in increased nuclei per field compared to cycloheximide-containing cultures. Additionally, infection rates in the presence of cycloheximide were approximately twice those observed in the absence of cycloheximide.

By IF microscopy, $A B$ were easily observed in all inclusions in $0 \mathrm{hpi}$ penicillin-exposed infected cells, whether cycloheximide was included (Figure 2(a)) or excluded (Figure 2(b)). Corresponding mock-exposed infected cells contained no $\mathrm{AB}$, regardless of the inclusion/exclusion of cycloheximide. Confirmatory TEM analysis (Figure 2(a)) demonstrated that the $\mathrm{AB}$ observed by IF microscopy were at least $2 \mu \mathrm{m}$ in diameter, as is typical for $\mathrm{AB}$, and that mock-exposed controls included $\mathrm{EB}$ and $\mathrm{RB}$ of normal size and morphology [34]. Titration by subpassage was used to evaluate infectious EB production, measured as inclusionforming units (IFU) per $\mathrm{mL}$. Mock-exposed inclusions cultured in the presence of cycloheximide yielded $7.39 \times$ $10^{7} \mathrm{IFU} / \mathrm{mL}$ (per well of cells), while 0 hpi penicillin-exposed inclusions yielded $5.43 \times 10^{3} \mathrm{IFU} / \mathrm{mL}$ (Figure $2(\mathrm{a})$ ). In the absence of cycloheximide, mock-exposed inclusions yielded $4.42 \times 10^{6} \mathrm{IFU} / \mathrm{mL}$, while 0 hpi penicillin-exposed inclusions yielded $1.25 \times 10^{1} \mathrm{IFU} / \mathrm{mL}$ (Figure $2(\mathrm{~b})$ ). Inclusion of cycloheximide resulted in increased IFU/mL in mock-exposed controls compared to exclusion of cycloheximide, as expected given the increased infection rate of host cells also observed. However, the observed penicillin-dependent decreases in
IFU/mL constituted a similar reduction to $<1 \%$ of the mockexposed control whether or not cycloheximide was included.

To determine the ability of $C$. pecorum to recover from penicillin exposure, infected cells were exposed at $0 \mathrm{hpi}$ until $35 \mathrm{hpi}$, when the medium was changed and exposure was continued or discontinued (recovery) for $24 \mathrm{~h}$ or $48 \mathrm{~h}$ (Figure 1). Cycloheximide exposure was concomitantly continued (Figure 3(a)) or excluded (Figures 3(b) and 3(c)) for the duration of incubation. Upon $24 \mathrm{~h}$ or $48 \mathrm{~h}$ of continued culture, significant Chlamydia-induced cell death occurred in mock-exposed controls, while cell survival was comparatively improved in 0 hpi penicillin-exposed groups, regardless of inclusion/exclusion of cycloheximide. In the presence of cycloheximide, mean nuclei per field values ranged from 17 to 20 for $0 \mathrm{hpi}$ penicillin-exposed cells, upon both $24 \mathrm{~h}$ and $48 \mathrm{~h}$ of continued culture, while mock-exposed controls retained only 1-3 nuclei per field (Figure 3(a)). In the absence of cycloheximide, mean nuclei per field values were larger and ranged from 47 to 52 for 0 hpi penicillin-exposed cells at 24 and $48 \mathrm{~h}$ of further culture, while mock-exposed cells retained 12-25 nuclei per field (Figure 3(b)). Therefore, for $24 \mathrm{~h}$ and $48 \mathrm{~h}$ continued culture, penicillin $\mathrm{G}$ exposure significantly protected HeLa cells from C. pecorum 1710 S-induced cell death independent of cycloheximide inclusion/exclusion. Thus, cells remained present in both cycloheximide-exposed and unexposed HeLa monolayers as a source of constituents/metabolites for potentially recovering Chlamydiae.

Infectious EB production (IFU/mL) increased upon discontinuation of $0 \mathrm{hpi}$-initiated penicillin exposure, compared to continued penicillin exposure, for both cycloheximideexposed infected cells (Table 1 ) and unexposed HeLa/C. pecorum (Figure 3(c)). Although the statistical significance of these changes was inconsistent due to high variability, the observed changes were generally substantial. Approximately 600- to 5000-fold increases for 0 hpi penicillin exposure were observed when comparing discontinued exposure (recovery) compared to continued penicillin exposure, in the presence of cycloheximide, at $24 \mathrm{~h}$ of continued culture (Table 1 ). 

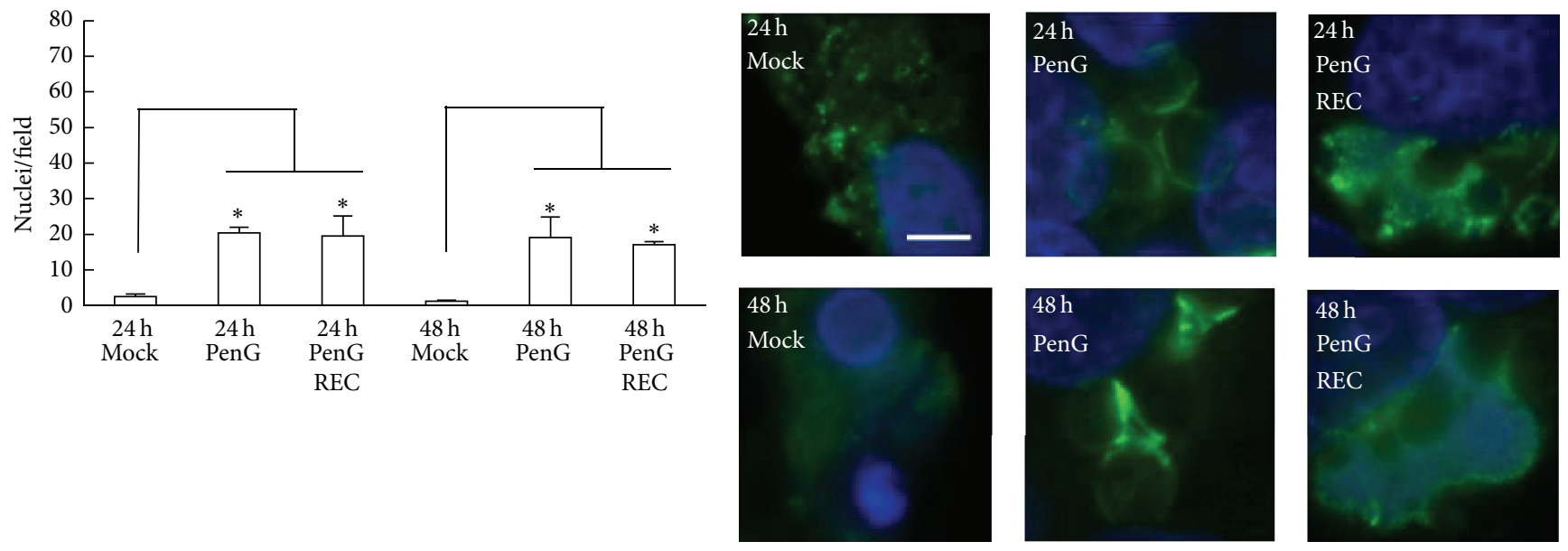

(a)
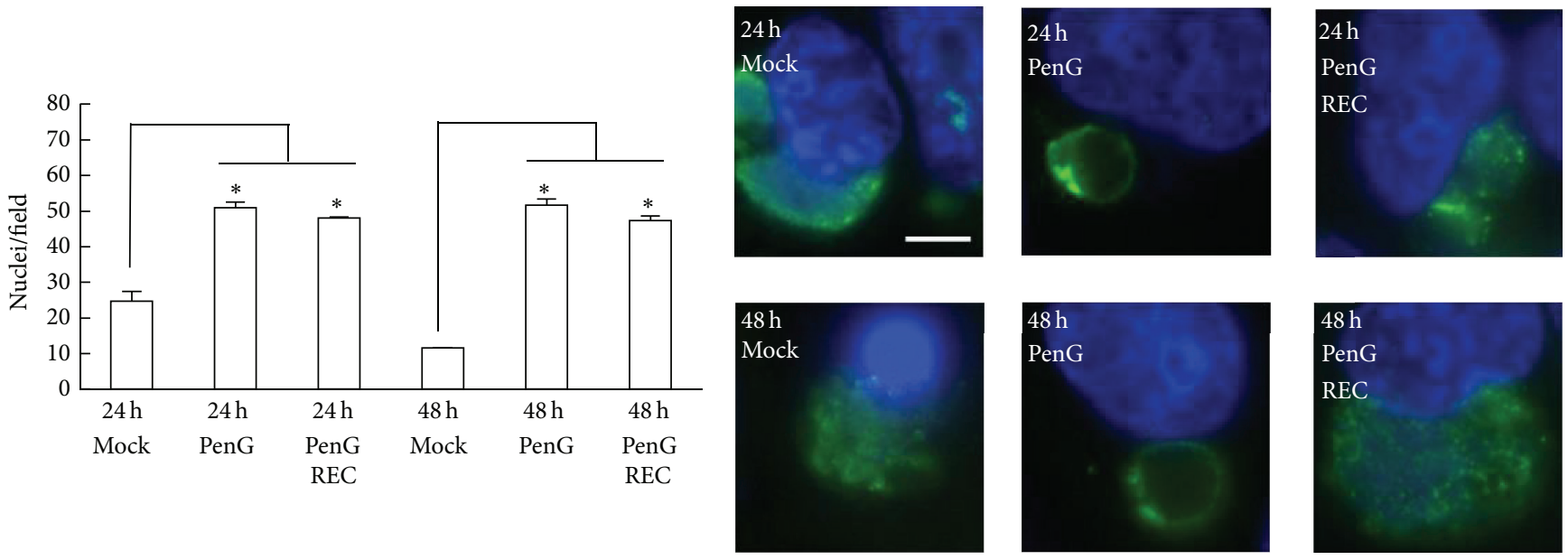

(b)

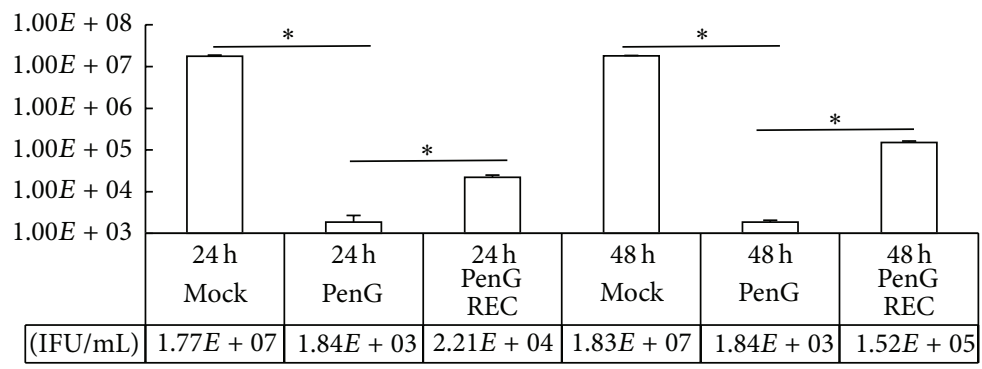

(c)

FIgURE 3: Penicillin G exposure prevents Chlamydia pecorum-mediated cell death and C. pecorum recovers infectivity upon discontinuation of penicillin exposure. C. pecorum-infected HeLa cells were penicillin G (PenG) exposed or diluent exposed (mock) from 0 to 35 hours postinfection (hpi). Cycloheximide was included (a) or not included (b, c) in the incubation medium. At 35 hpi, medium was changed to continue or discontinue (recovery, REC) penicillin exposure for 24 or 48 hours (h) and cycloheximide was maintained for (a). Inclusions were visualized and mean nuclei per field were determined as described for Figure 2. Representative immunofluorescence microscopic images illustrate cell death in mock-exposed controls, inclusions containing aberrant bodies upon continued penicillin exposure and normalappearing inclusions upon recovery (REC). Production of infectious elementary bodies was determined by titration by subpassage and expressed as inclusion-forming units (IFU)/mL. Results are means \pm standard deviation. The two-tailed $t$-test was used to compare means; $p$ $\leq 0.05=\operatorname{significant}\left({ }^{*}\right) ; n=2$. Scale bars $=5 \mu \mathrm{m}$. 
Similarly, but to a greater extent, approximately 30,000 - to 340,000 -fold increases in IFU/mL of 0 hpi penicillin-exposed groups were observed in cycloheximide-including recovery conditions, compared to continued exposure upon $48 \mathrm{~h}$ of continued culture (Table 1). In the absence of cycloheximide, results were similar but more moderate in nature. At $24 \mathrm{~h}$ of continued culture, discontinuation of penicillin exposure resulted in a 12 -fold increase in IFU/ $\mathrm{mL}$ compared to continued exposure, while, at $48 \mathrm{~h}$ of continued culture, an 82 -fold increase in IFU/mL was observed in discontinued penicillin exposure versus continued exposure (Figure 3(c)). Thus, the inclusion of cycloheximide potentiated a more robust recovery from penicillin $\mathrm{G}$ exposure. However, either inclusion or exclusion of cycloheximide allowed increased infectious $C$. pecorum EB production upon discontinuation of penicillin exposure. Furthermore, regardless of the inclusion/exclusion of cycloheximide, increased recovery time from $24 \mathrm{~h}$ to $48 \mathrm{~h}$ of continued culture was associated with increased infectious EB production.

AB were consistently observed by IF microscopy upon continued penicillin exposure, regardless of the duration of exposure or the presence (Figure 3(a)) or absence (Figure 3(b)) of cycloheximide in the assay. Some, but not all, recovery group inclusions showed areas of denser, more granular LPS-positive staining, consistent with the appearance of mock-exposed inclusions at $35 \mathrm{hpi}$ (Figures 3(a) and 3(b) right panels, compared to Figures 2(a) and 2(b) left panels). These inclusions were frequently associated with shrunken, brightly staining host cell nuclei, indicating that as inclusions became more normal in appearance, $C$. pecorum $1710 \mathrm{~S}$ was still capable of exerting its cytotoxic effect on HeLa cells. Mock-exposed cells showed no AB, at $24 \mathrm{~h}$ or $48 \mathrm{~h}$ of continued culture, regardless of inclusion/exclusion of cycloheximide or duration of incubation (Figures 3(a) and 3(b)).

Initiation of penicillin $\mathrm{G}$ exposure at $14 \mathrm{hpi}$ (see Figure 1), in the presence of cycloheximide, was carried out to determine if Chlamydiae being at the stage of early RB development upon exposure might modulate the described effects of penicillin $G$ exposure or subsequent continuation/discontinuation of this exposure on Chlamydiae. During 0 hpi penicillin $\mathrm{G}$ exposure, EB are exposed to penicillin as they enter the host cells and initiate inclusion formation. However, 14 hpi addition of penicillin postpones the exposure of the Chlamydiae until EB have differentiated into RB, and early $\mathrm{RB}$ replication/development is starting or will soon start, but redifferentiation into EB has not begun [36]. Thus, upon $T_{14}$ exposure, intracellular RB are the bacterial forms initially exposed to penicillin.

We found, by IF microscopy and TEM (not shown), that 14 hpi penicillin G-exposed HeLa/C. pecorum had normal inclusions in mock-exposed groups and AB-containing inclusions in $14 \mathrm{hpi}$ penicillin-exposed groups at $35 \mathrm{hpi}$ and at $24 \mathrm{~h}$ or $48 \mathrm{~h}$ of continued exposure, similar to the $0 \mathrm{hpi}$ penicillin exposure results. Later initiation of exposure was associated with somewhat larger numbers (usually 6 to 8 $A B$, versus 1-2 $A B$ per inclusion) of slightly smaller $A B$ (though still at least $2 \mu \mathrm{m}$ in diameter). Upon $14 \mathrm{hpi}$ penicillin exposure, the mean nuclei per field value was 24 and the host cell infection rate was $51 \%$ at 35 hpi. For 14 hpi initiation of penicillin exposure and subsequent continuation or discontinuation of penicillin exposure, mean nuclei per field values at $24 \mathrm{~h}$ and $48 \mathrm{~h}$ of continued culture ranged from 16 to 22 and 15 to 16 , respectively. These $35 \mathrm{hpi}$ and $24 / 48 \mathrm{~h}$ data are statistically equivalent to those observed for $0 \mathrm{hpi}$ penicillin exposure. Additionally, upon $14 \mathrm{hpi}$ initiation of penicillin exposure, the IFU/mL value at $35 \mathrm{hpi}\left(2.08 \times 10^{4} \mathrm{IFU} / \mathrm{mL}\right)$ was less than $1 \%$ of the mock-exposed control value, similar to the 0 hpi results. Furthermore, IFU/mL values of discontinued penicillin exposure versus continued penicillin exposure (see Table 1) were very similar regardless of time of initiation of penicillin exposure. Later initiation of exposure, and thus decreased total penicillin exposure time, was not correlated with increased infectious EB production (IFU/mL).

Beta lactams have long been considered nonbactericidal against the Chlamydiae, instead inducing, in vitro, a reversible state of bacterial stress, termed persistence or chlamydial stress. The original report of in vitro chlamydial persistence [16] described aberrant bacterial forms and reduced infectivity upon penicillin $\mathrm{G}$ exposure and demonstrated the reversibility that defines chlamydial persistence, a phenomenon now known to be inducible by various stressors including nutrient deprivation, host interferon gamma production, and coinfection [30]. The in vitro antichlamydial effect of beta lactams has also been reported as bactericidal under some conditions, namely, exposure to penicillin early after chlamydial infection and specifically in the absence of pharmacologically (cycloheximide) inhibited host de novo protein synthesis [35]. Chlamydiae were thought to lack peptidoglycan, the target of beta lactams, so the response of Chlamydiae to beta lactams has been anomalous [37]. A recent report providing evidence of chlamydial peptidoglycan [38] clarifies the antichlamydial effect of beta lactams and emphasizes the role they may play in modulation of natural infections.

In this study, experiments were primarily designed to facilitate C. pecorum recovery from penicillin to determine if this species can, under conditions permissive for persistence induction, exhibit the hallmarks of persistence. In a previous report, exclusion of cycloheximide was sufficient to render C. trachomatis incapable of recovery from 1,10 , or $100 \mathrm{U} / \mathrm{mL}$ penicillin $\mathrm{G}$ exposure, while recovery from $100 \mathrm{U} / \mathrm{mL}$ penicillin $\mathrm{G}$ was possible in the presence of cycloheximide [35]. Thus, we included cycloheximide in our experiments but also compared the effects to those elicited in the absence of cycloheximide. We found that although cycloheximide potentiated the recovery of C. pecorum 1710 S infectious EB production upon the removal of penicillin $G$, it was not required for such recovery. In vivo, local modulation of host protein synthesis may occur via mechanisms in response to chlamydial infection and/or infection with other bacteria or viruses. These may include immune-mediated interferondependent tryptophan reduction [29] or specific antiviral responses [39], for example. Therefore, though cycloheximide may be considered to limit the biological relevance of in vitro models of chlamydial infection, reduced host protein translation may impact chlamydial entry into and exit from the chlamydial stress response in vivo. 
HeLa cells, being human-derived cells, do not represent a natural host species for C. pecorum. However, previous reports indicate that penicillin $G$ exposure of $C$. trachomatisinfected HeLa cells, both in the presence [35] and the absence [22] of cycloheximide, induces an antichlamydial effect consistent with persistence. Additionally, recent work in our laboratory [32] showed that damage associated molecular patterns (DAMP) exposure of C. pecorum-infected cells also elicits an antichlamydial effect in HeLa cells that is consistent with persistence.

The concentration of penicillin G used in this study was chosen based on preliminary optimization (not shown) of the induction of $\mathrm{AB}$ formation in various chlamydial species and host cells lines, in which $1-100 \mathrm{U} / \mathrm{mL}$ consistently yielded AB in all inclusions. While studies evaluating penicillin-induced persistence frequently use hundreds of $\mathrm{U} / \mathrm{mL}$, a recent in vitro study showed penicillin $\mathrm{G}$ reduced C. trachomatis infectious $\mathrm{EB}$ production by $>99 \%$ at $0.02 \mathrm{U} / \mathrm{mL}$ [22]. Thus, we chose a concentration not in substantial excess of that required to induce C. pecorum $1710 \mathrm{~S} \mathrm{AB}$ formation and to reduce infectious $\mathrm{EB}$ production. $1 \mathrm{U} / \mathrm{mL}$ penicillin $\mathrm{G}$ induced $\mathrm{AB}$ formation in all inclusions and reduced infectious EB production to $<1 \%$ of control values. Earlier exposure did not result in statistically reduced infectious EB production. Furthermore, later exposure, resulting in more $\mathrm{AB}$ per inclusion but a similar number of inclusions compared to early exposure or mock exposure, did not increase recovery of infectious EB production upon cessation of penicillin exposure.

Chlamydial species is also likely to impact the effect of beta lactams both in vitro and in vivo. We recently demonstrated that C. pecorum is more capable of recovering from DAMP-induced reduced infectious EB production than $C$. trachomatis [32]; this suggests that $C$. pecorum may be more able to recover from persistence induced by various stressors than C. trachomatis. Human strains of C. pneumoniae are more able to recover from penicillin-induced persistence than animal strains [40], suggesting that species- and strainspecific sensitivity to stressors, including antibiotics, may be common within the Chlamydiae. Experimental parameters such as specific beta lactam drug used, timing of initiation exposure and duration of exposure, and timing of removal of drug for recovery and duration of recovery, as well as differences in measurement/determination of infectivity could be expected to impact degree and detection of entry into and recovery from beta lactam-induced chlamydial stress/persistence. Such factors, as well as biological variation amongst the Chlamydiae, are also likely to contribute to differing reports of bactericidal and bacteriostatic effects of beta lactam antibiotics amongst the Chlamydiae.

Questions remain concerning the biological importance of chlamydial persistence/stress in vivo, especially in naturally occurring infections. While aberrant chlamydial forms have been demonstrated in porcine [41] and human [42] specimens, veterinary/medical consequences are debated [23]. Results from a recent mouse model study demonstrated that beta lactam-induced persistent chlamydial genital infection is more prone to azithromycin treatment failure than productive chlamydial infection [21]. Intestinal chlamydial infection in mice has also been demonstrated to be more resistant to azithromycin than genital infection [43]. Asymptomatic gastrointestinal $C$. pecorum infections and other such chlamydial infections are common and are subject to beta lactam exposure due to widespread agricultural use of these drugs. Thus, beta lactam therapy is likely to expose undetected Chlamydiae to persistence inducers. While it is known that persistent/stressed Chlamydiae exhibit altered protein expression in vitro compared to nonstressed controls [44-48], the potential effect of $\mathrm{AB} /$ chlamydial stress on porcine abortion rates, failure to gain weight, or other clinical symptoms is entirely unknown but interesting and potentially important.

Recent clinical findings suggest that asymptomatic human gastrointestinal chlamydial infection also occurs [4951]. These data and clinical findings suggest that difficulties in elimination of chlamydial infection may be common to both humans and other animals and may be modulated by beta lactam exposure. In a recent porcine-specific example of failure to eliminate chlamydial infection with standard antimicrobial therapy, asymptomatic chlamydial intestinal infection in multiple pig herds in Switzerland remained present independent of antibiotic treatments, including tetracycline treatment [10].

\section{Conclusions}

We report that, as hypothesized, C. pecorum 1710 S enters a state of chlamydial/stress persistence upon beta lactam exposure. However, the extensive biological and genetic diversity of C. pecorum, including various clinical presentations and serological characteristics, in vitro growth characteristics, and genetic characteristics such as the recently demonstrated differential presence of a novel plasmid [4, 52-54], suggests that the wide range of strains comprising this species warrant continued scrutiny. Since the effect of asymptomatic chlamydial infections on long-term fitness of animals and humans and the role of chlamydial stress/persistence under such circumstances is unknown, the effects of beta lactams on the Chlamydiae are of continued veterinary and medical interest.

\section{Conflict of Interests}

The authors declare that there is no conflict of interests regarding the publication of this paper.

\section{Acknowledgments}

HeLa cells were kindly provided by Christian Blenn, University of Zurich. This work was supported by 310030_147026, http://www.snf.ch/. The authors thank Lisbeth Nufer, University of Zurich, for excellent technical assistance with all electron microscopy.

\section{References}

[1] H. Fukushi and K. Hirai, "Chlamydia pecorum-the fourth species of genus Chlamydia," Microbiology and Immunology, vol. 37, no. 7, pp. 516-522, 1993. 
[2] K. Schautteet and D. Vanrompay, "Chlamydiaceae infections in pig," Veterinary Research, vol. 42, no. 1, article 29, 2011.

[3] B. Kaltenboeck and J. Storz, "Biological properties and genetic analysis of the ompA locus in chlamydiae isolated from swine," American Journal of Veterinary Research, vol. 53, no. 9, pp. 14821487, 1992.

[4] P. Spears and J. Storz, "Biotyping of Chlamydia psittaci based on inclusion morphology and response to diethylaminoethyldextran and cycloheximide," Infection and Immunity, vol. 24, no. 1, pp. 224-232, 1979.

[5] F. Guscetti, Chlamydia in Swine: Field Studies and Experimental Enteric Infections in Gnotobiotic Piglets, Institute for Veterinary Pathology, University of Zurich, Zürich, Switzerland, 1999.

[6] R. Thoma, F. Guscetti, I. Schiller, N. Schmeer, L. Corboz, and A. Pospischil, "Chlamydiae in porcine abortion," Veterinary Pathology, vol. 34, no. 5, pp. 467-469, 1997.

[7] I. Schiller, R. Koesters, R. Weilenmann et al., "Mixed infections with porcine Chlamydia trachomatis/pecorum and infections with ruminant Chlamydia psittaci serovar 1 associated with abortions in swine," Veterinary Microbiology, vol. 58, no. 2-4, pp. 251-260, 1997.

[8] A. Poudel, T. H. Elsasser, K. S. Rahman, E. U. Chowdhury, and B. Kaltenboeck, "Asymptomatic endemic Chlamydia pecorum infections reduce growth rates in calves by up to 48 percent," PLoS ONE, vol. 7, no. 9, Article ID e44961, 2012.

[9] I. Chopra and M. Roberts, "Tetracycline antibiotics: mode of action, applications, molecular biology, and epidemiology of bacterial resistance," Microbiology and Molecular Biology Reviews, vol. 65, no. 2, pp. 232-260, 2001.

[10] K. Hoffmann, F. Schott, M. Donati et al., "Prevalence of chlamydial infections in fattening pigs and their influencing factors," PLoS ONE, vol. 10, no. 11, Article ID e0143576, 2015.

[11] N. Borel, N. Regenscheit, A. Di Francesco et al., "Selection for tetracycline-resistant Chlamydia suis in treated pigs," Veterinary Microbiology, vol. 156, no. 1-2, pp. 143-146, 2012.

[12] C. A. Macarov, L. Tong, M. Martínez-Huélamo et al., "Multi residue determination of the penicillins regulated by the European Union, in bovine, porcine and chicken muscle, by LCMS/MS," Food Chemistry, vol. 135, no. 4, pp. 2612-2621, 2012.

[13] M. Li, R. Gehring, L. Tell, R. Baynes, Q. Huang, and J. E. Riviere, "Interspecies mixed-effect pharmacokinetic modeling of penicillin G in cattle and swine," Antimicrobial Agents and Chemotherapy, vol. 58, no. 8, pp. 4495-4503, 2014.

[14] S. J. Lupton, W. L. Shelver, D. J. Newman, S. Larsen, and D. J. Smith, "Depletion of penicillin G residues in heavy sows after intramuscular injection. Part I: tissue residue depletion," Journal of Agricultural and Food Chemistry, vol. 62, no. 30, pp. 75777585, 2014.

[15] W. L. Shelver, S. J. Lupton, D. J. Newman, S. Larsen, and D. J. Smith, "Depletion of penicillin G residues in heavy sows after intramuscular injection. Part II. Application of kidney inhibition swab tests," Journal of Agricultural and Food Chemistry, vol. 62, no. 30, pp. 7586-7592, 2014.

[16] A. Matsumoto and G. P. Manire, "Electron microscopic observations on the effects of penicillin on the morphology of Chlamydia psittaci," Journal of Bacteriology, vol. 101, no. 1, pp. 278-285, 1970.

[17] J. Peters, S. Hess, K. Endlich et al., "Silencing or permanent activation: host-cell responses in models of persistent Chlamydia pneumoniae infection," Cellular Microbiology, vol. 7, no. 8, pp. 1099-1108, 2005.
[18] W. M. Huston, C. Theodoropoulos, S. A. Mathews, and P. Timms, "Chlamydia trachomatis responds to heat shock, penicillin induced persistence, and IFN-gamma persistence by altering levels of the extracytoplasmic stress response protease HtrA," BMC Microbiology, vol. 8, no. 1, article 190, 2008.

[19] R. Phillips Campbell, J. Kintner, J. Whittimore, and R. V. Schoborg, "Chlamydia muridarum enters a viable but noninfectious state in amoxicillin-treated BALB/c mice," Microbes and Infection, vol. 14, no. 13, pp. 1177-1185, 2012.

[20] V. A. Ong, J. W. Marsh, A. Lawrence, J. A. Allan, P. Timms, and W. M. Huston, "The protease inhibitor JO146 demonstrates a critical role for CtHtrA for Chlamydia trachomatis reversion from penicillin persistence," Frontiers in Cellular and Infection Microbiology, vol. 3, article 100, 2013.

[21] R. Phillips-Campbell, J. Kintner, and R. V. Schoborg, "Induction of the Chlamydia muridarum stress/persistence response increases azithromycin treatment failure in a murine model of infection," Antimicrobial Agents and Chemotherapy, vol. 58, no. 3, pp. 1782-1784, 2014.

[22] J. Kintner, D. Lajoie, J. Hall, J. Whittimore, and R. V. Schoborg, "Commonly prescribed $\beta$-lactam antibiotics induce C. trachomatis persistence/stress in culture at physiologically relevant concentrations," Frontiers in Cellular and Infection Microbiology, vol. 4, article 44, 2014.

[23] P. M. Bavoil, "What's in a word: the use, misuse, and abuse of the word 'persistence' in Chlamydia biology," Frontiers in Cellular and Infection Microbiology, vol. 4, article 27, 2014.

[24] P. B. Wyrick and S. T. Knight, "Pre-exposure of infected human endometrial epithelial cells to penicillin in vitro renders Chlamydia trachomatis refractory to azithromycin," Journal of Antimicrobial Chemotherapy, vol. 54, no. 1, pp. 79-85, 2004.

[25] R. J. Hogan, S. A. Mathews, S. Mukhopadhyay, J. T. Summersgill, and P. Timms, "Chlamydial persistence: beyond the biphasic paradigm," Infection and Immunity, vol. 72, no. 4, pp. 1843-1855, 2004.

[26] Pudjiatmoko, H. Fukushi, Y. Ochiai, T. Yamaguchi, and K. Hirai, "In vitro susceptibility of Chlamydia pecorum to macrolides, tetracyclines, quinolones and $\beta$-lactam," Microbiology and Immunology, vol. 42, no. 1, pp. 61-63, 1998.

[27] L. A. Black, D. P. Higgins, and M. Govendir, "In vitro activity of chloramphenicol, florfenicol and enrofloxacin against Chlamydia pecorum isolated from koalas (Phascolarctos cinereus)," Australian Veterinary Journal, vol. 93, no. 11, pp. 420-423, 2015.

[28] W. L. Beatty, R. P. Morrison, and G. I. Byrne, "Persistent chlamydiae: from cell culture to a paradigm for chlamydial pathogenesis," Microbiological Reviews, vol. 58, no. 4, pp. 686699, 1994.

[29] R. V. Schoborg, "Chlamydia persistence-a tool to dissect chlamydia-host interactions," Microbes and Infection, vol. 13, no. 7, pp. 649-662, 2011.

[30] P. B. Wyrick, "Chlamydia trachomatis persistence in vitro: an overview," Journal of Infectious Diseases, vol. 201, supplement 2, pp. S88-S95, 2010.

[31] N. Borel, C. Dumrese, U. Ziegler, A. Schifferli, C. Kaiser, and A. Pospischil, "Mixed infections with Chlamydia and porcine epidemic diarrhea virus-a new in vitro model of chlamydial persistence," BMC Microbiology, vol. 10, no. 1, article 201, 2010.

[32] C. A. Leonard, R. V. Schoborg, N. Borel, and B. Kaltenboeck, "Damage/danger associated molecular patterns (DAMPs) modulate Chlamydia pecorum and C. trachomatis serovar E inclusion development in vitro," PLoS ONE, vol. 10, no. 8, Article ID e0134943, 2015. 
[33] S. Deka, J. Vanover, S. Dessus-Babus et al., "Chlamydia trachomatis enters a viable but non-cultivable (persistent) state within herpes simplex virus type 2 (HSV-2) co-infected host cells," Cellular Microbiology, vol. 8, no. 1, pp. 149-162, 2006.

[34] H. Marti, M. Koschwanez, T. Pesch, C. Blenn, and N. Borel, "Water-filtered infrared a irradiation in combination with visible light inhibits acute chlamydial infection," PLOS ONE, vol. 9, no. 7, Article ID e102239, 2014.

[35] M. Dumoux, S. M. Le Gall, M. Habbeddine et al., "Penicillin kills Chlamydia following the fusion of bacteria with lysosomes and prevents genital inflammatory lesions in C. muridaruminfected mice," PLoS ONE, vol. 8, no. 12, Article ID e83511, 2013.

[36] Y. M. AbdelRahman and R. J. Belland, "The chlamydial developmental cycle," FEMS Microbiology Reviews, vol. 29, no. 5, pp. 949-959, 2005.

[37] J. W. Moulder, "Why is Chlamydia sensitive to penicillin in the absence of peptidoglycan?" Infectious Agents and Disease, vol. 2, no. 2, pp. 87-99, 1993.

[38] G. W. Liechti, E. Kuru, E. Hall et al., "A new metabolic cell-wall labelling method reveals peptidoglycan in Chlamydia trachomatis," Nature, vol. 506, no. 7489, pp. 507-510, 2014.

[39] D. Walsh, M. B. Mathews, and I. Mohr, "Tinkering with translation: protein synthesis in virus-infected cells," Cold Spring Harbor Perspectives in Biology, vol. 5, no. 1, Article ID a012351, 2013.

[40] A. Chacko, K. W. Beagley, P. Timms, and W. M. Huston, "Human Chlamydia pneumoniae isolates demonstrate ability to recover infectivity following penicillin treatment whereas animal isolates do not," FEMS Microbiology Letters, vol. 362, no. 6, Article ID fnv015, 2015.

[41] A. Pospischil, N. Borel, E. H. Chowdhury, and F. Guscetti, "Aberrant chlamydial developmental forms in the gastrointestinal tract of pigs spontaneously and experimentally infected with Chlamydia suis," Veterinary Microbiology, vol. 135, no. 1-2, pp. 147-156, 2009.

[42] N. Borel, J. T. Summersgill, S. Mukhopadhyay, R. D. Miller, J. A. Ramirez, and A. Pospischil, "Evidence for persistent Chlamydia pneumoniae infection of human coronary atheromas," Atherosclerosis, vol. 199, no. 1, pp. 154-161, 2008.

[43] L. Yeruva, S. Melnyk, N. Spencer, A. Bowlin, and R. G. Rank, "Differential susceptibilities to azithromycin treatment of chlamydial infection in the gastrointestinal tract and cervix," Antimicrobial Agents and Chemotherapy, vol. 57, no. 12, pp. 6290-6294, 2013.

[44] P. Timms, D. Good, C. Wan et al., "Differential transcriptional responses between the interferon- $\gamma$-induction and ironlimitation models of persistence for Chlamydia pneumoniae," Journal of Microbiology, Immunology and Infection, vol. 42, no. 1, pp. 27-37, 2009.

[45] A. Klos, J. Thalmann, J. Peters, H. C. Gérard, and A. P. Hudson, "The transcript profile of persistent Chlamydophila (Chlamydia) pneumoniae in vitro depends on the means by which persistence is induced," FEMS Microbiology Letters, vol. 291, no. 1, pp. 120126, 2009.

[46] A. Kokab, R. Jennings, A. Eley, A. A. Pacey, and N. A. Cross, "Analysis of modulated gene expression in a model of interferon- $\gamma$-induced persistence of Chlamydia trachomatis in HEp-2 cells," Microbial Pathogenesis, vol. 49, no. 5, pp. 217-225, 2010.
[47] Y. M. AbdelRahman, L. A. Rose, and R. J. Belland, "Developmental expression of non-coding RNAs in Chlamydia trachomatis during normal and persistent growth," Nucleic Acids Research, vol. 39, no. 5, pp. 1843-1854, 2011.

[48] M. Di Pietro, A. Tramonti, F. De Santis et al., "Analysis of gene expression in penicillin $\mathrm{G}$ induced persistence of Chlamydia pneumoniae," Journal of Biological Regulators and Homeostatic Agents, vol. 26, no. 2, pp. 277-284, 2012.

[49] A. Ding and R. Challenor, "Rectal Chlamydia in heterosexual women: more questions than answers," International Journal of STD \& AIDS, vol. 25, no. 8, pp. 587-592, 2014.

[50] G. A. F. S. van Liere, C. J. P. A. Hoebe, P. F. G. Wolffs, and N. H. T. M. Dukers-Muijrers, "High co-occurrence of anorectal chlamydia with urogenital chlamydia in women visiting an STI clinic revealed by routine universal testing in an observational study; a recommendation towards a better anorectal chlamydia control in women," BMC Infectious Diseases, vol. 14, no. 1, article 274, 2014.

[51] J. S. Hocking, F. Y. S. Kong, P. Timms, W. M. Huston, and S. N. Tabrizi, "Treatment of rectal chlamydia infection may be more complicated than we originally thought," Journal of Antimicrobial Chemotherapy, vol. 70, no. 4, pp. 961-964, 2015.

[52] J. A. Perez-Martinez and J. Storz, "Antigenic diversity of Chlamydia psittaci of mammalian origin determined by microimmunofluorescence," Infection and Immunity, vol. 50, no. 3, pp. 905-910, 1985.

[53] M. Jelocnik, N. L. Bachmann, B. Kaltenboeck et al., "Genetic diversity in the plasticity zone and the presence of the chlamydial plasmid differentiates Chlamydia pecorum strains from pigs, sheep, cattle, and koalas," BMC Genomics, vol. 16, no. 1, article 893, 2015.

[54] N. L. Bachmann, M. J. Sullivan, M. Jelocnik, G. S. Myers, P. Timms, and A. Polkinghorne, "Culture-independent genome sequencing of clinical samples reveals an unexpected heterogeneity of infections by Chlamydia pecorum," Journal of Clinical Microbiology, vol. 53, no. 5, pp. 1573-1581, 2015. 

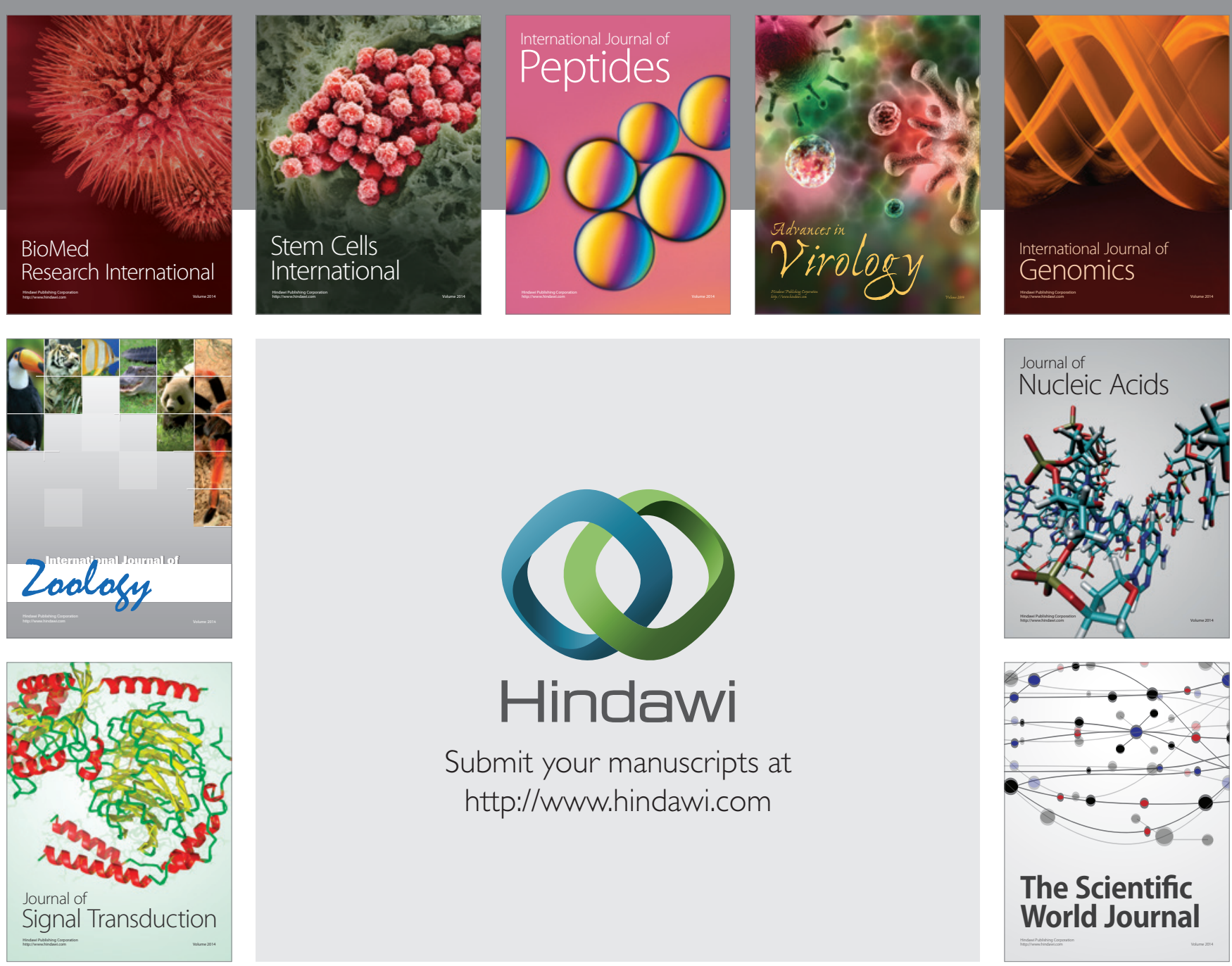

Submit your manuscripts at

http://www.hindawi.com
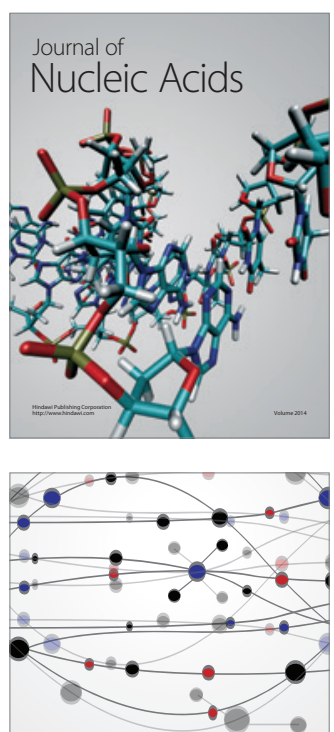

The Scientific World Journal
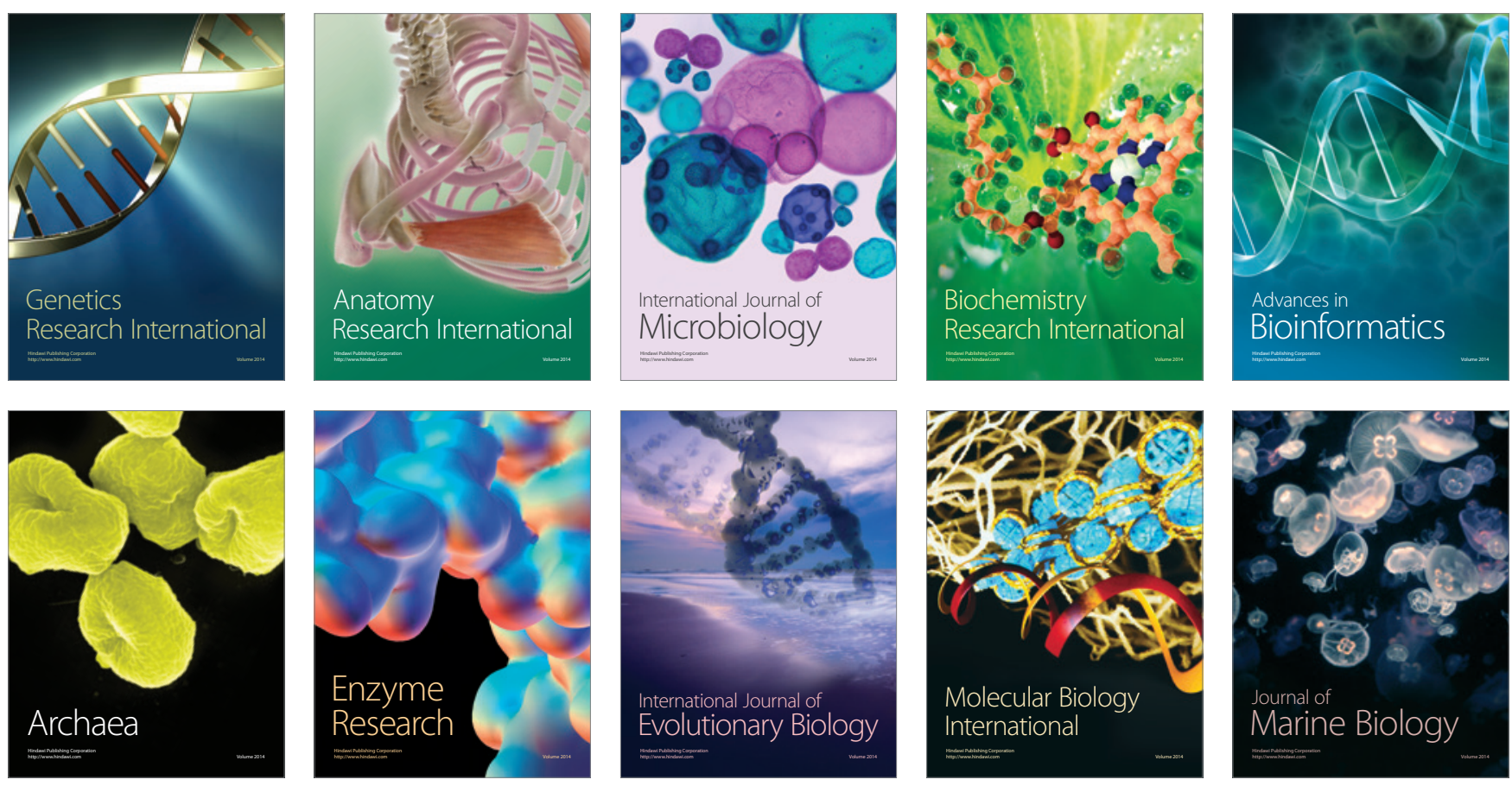